La consulta previa de la construcción y mantenimiento de la infraestructura de los servicios públicos beneficiosa para los pueblos indígenas

Iván Lanegra Quispe

En el presente artículo, el autor analiza

el supuesto recogido en el Reglamento de la Ley de Consulta Previa que fija los criterios para determinar cuándo la construcción y mantenimiento de infraestructura en materia de salud, educación, así como la provisión de servicios públicos, resultan beneficiosas para los pueblos indígenas. A su vez, el autor evalúa su tratamiento en la directiva emitida por el Ministerio de Cultura y brinda alternativas de regulación para atender el caso planteado.

Abogado por la Pontificia Universidad Católica del Perú y egresado de la Maestría en Ciencia Política de la misma universidad. Profesor de Ciencia Política en la Universidad del Pacífico y de la Especialidad de Ciencia Política y Gobierno, así como del Diploma de Especialización en Derecho Ambiental y de los Recursos Naturales en la Pontificia Universidad Católica del Perú. Ha sido Adjunto para el Medio Ambiente, Servicios Públicos y Pueblos Indígenas de la Defensoría del Pueblo y Viceministro de Interculturalidad. 


\section{La consulta previa de la construcción y mantenimiento de la infraestructura de los servicios públicos beneficiosa para los pueblos indígenas}

Hacer efectivo el derecho a la consulta previa que tienen los pueblos indígenas constituye una de los principales desafíos de la política indígena en el Perú y en América Latina. La consulta constituye -junto a la participación- uno de los pilares del Convenio 169 de la Organización Internacional del Trabajo -OIT. De acuerdo con el artículo $6^{\circ}$ de dicho tratado internacional los Estados parte deben consultar a los pueblos indígenas, "mediante procedimientos apropiados y en particular a través de sus instituciones representativas, cada vez que se prevean medidas legislativas 0 administrativas susceptibles de afectarles directamente".

En el Perú, la aprobación de la Ley de Consulta Previa ${ }^{1}$ y su reglamento ${ }^{2}$ establecieron los procedimientos y mecanismos institucionales que permitirían la implementación de los procesos de consulta. La citada Ley introdujo un conjunto de principios orientadores que deben servir para una adecuada aplicación de dicha norma. Uno de estos principios es de la flexibilidad, el cual señala que la consulta "(...) debe desarrollarse mediante procedimientos apropiados al tipo de medida legislativa 0 administrativa que se busca adoptar, así como tomando en cuenta las circunstancias y características especiales de los pueblos indígenas u originarios involucrados". ${ }^{3}$

Es en dicho marco que el Reglamento de la Ley de Consulta introdujo una disposición especial. De acuerdo con ella,

"La construcción y mantenimiento de infraestructura en materia de salud, educación, así como la necesaria para la provisión de servicios públicos que, en coordinación con los pueblos indígenas, esté orientada a beneficiarlos, no requerirán ser sometidos al procedimiento de consulta previsto en el Reglamento [de la Ley de Consulta Previa]".

Recientemente, el Ministerio de Cultura -responsable de conducir la política de consulta previa- aprobó los "Procedimientos para [la] aplicación de los dispuesto en la Décimo Quinta Disposición Complementaria, Transitoria y Final del Reglamento de la Ley 29785, Ley del Derecho a la Consulta Previa a los Pueblos Indígenas u Originarios,

$1 \quad$ Ley 29785, Ley del Derecho a la Consulta Previa a los Pueblos Indígenas u Originarios, reconocido en el Convenio 169 de la Organización Internacional del Trabajo (OIT), publicada el 7 de setiembre de 2011.

2 Decreto Supremo 001-2012-MC, Reglamento de la Ley 29785, Ley del Derecho a la Consulta Previa a los Pueblos Indígenas u Originarios, reconocido en el Convenio 169 de la Organización Internacional del Trabajo (OIT) publicado el 3 de abril de 2012.

3 Inciso d), artículo 4 de la Ley 29785.

4 Décimo Quinta Disposición Complementaria, Transitoria y Final del Decreto Supremo 0012012-MC. 
reconocido en el Convenio 169 de la Organización Internacional del Trabajo". ${ }^{5}$ El presente artículo analizará el supuesto recogido en el Reglamento de la Ley de Consulta y evaluará su tratamiento en la directiva emitida por el Ministerio de Cultura.

\section{Análisis de la Décimo Quinta Disposición Complementaria, Transitoria y Final del Reglamento de la Ley 29785}

Una de las principales cuestiones prácticas que debe asumir el Estado al implementar la consulta previa se deriva de la diversidad y cantidad de decisiones estatales medidas administrativas y legislativas- que se dictan a diario. ¿Cuáles de esas medidas deben ser consultadas previamente? Aquellas que puedan generar una afectación directa sobre los pueblos indígenas. De conformidad con el Reglamento de la Ley de Consulta, "(...) una medida legislativa o administrativa afecta directamente al o los pueblos indígenas cuando contiene aspectos que pueden producir cambios en la situación jurídica o en el ejercicio de los derechos colectivos de tales pueblos". ${ }^{6}$ Dicha afectación puede ser positiva o negativa ${ }^{7}$. No obstante, la norma no hace referencia alguna al grado de afectación involucrado. Esto podría originar que determinadas actividades estatales -que sin duda pueden afectar a los pueblos indígenas- cuyo número sea muy grande, genere una gran presión sobre las autoridades públicas, obligando a realizar miles de procesos de consulta cada año.

Es este supuesto el que recoge la Décimo Quinta Disposición Complementaria, Transitoria y Final del Reglamento de la Ley 29785. Cada año, el Estado está a cargo de la construcción y mantenimiento de escuelas y centros de salud en todo el país, así como de diversos servicios públicos (agua y saneamiento, electricidad, telecomunicaciones, recolección y disposición final de residuos sólidos, etc.). Cuando estas actividades se realizan en el ámbito de los pueblos indígenas, pueden originar afectaciones, en gran medida positivas, en tanto los beneficiarios de dichos servicios son los propios pueblos indígenas.

¿Cómo atender este supuesto? Una opción es aplicar el procedimiento establecido en el Reglamento de Consulta Previa. Sin embargo, esto podría originar un esfuerzo grande con efectos poco significativos sobre los derechos de los pueblos indígenas, distrayendo recursos que podrían utilizarse para las consultas en decisiones con mayor impacto. Es por dicha razón que el reglamento ha establecido una excepción indicando que el mantenimiento y construcción de los mencionados servicios "(...) no requerirán ser sometidos al procedimiento de consulta previsto en el Reglamento [de la Ley de Consulta Previa]." ¿Esto quiere decir que quedan exceptuados de la obligación general

5 Directiva 001-2016-VMI/MC, aprobada mediante la Resolución Viceministerial 103-2016VMI-MC, del 27 de mayo de 2016.

$6 \quad$ Inciso c) del artículo $4^{\circ}$ de la Ley 29785.

7 Ministerio de Cultura, Consulta a los Pueblos Indígenas: Guía Metodológica, Lima, 2013, p.7. 
de consultar? La respuesta es no. Es decir, si hay afectación con dichas actividades conforme la definición del propio Reglamento- queda configurado el deber estatal de consultar. Solo que no sería necesario acudir al procedimiento ordinario de consulta previsto en el Reglamento.

¿Qué procedimiento debe seguirse entonces? El Reglamento no lo indica. No obstante, la norma precisa que esta excepción solo puede aplicarse cuando "(...) en coordinación con los pueblos indígenas, esté orientada a beneficiarlos". La referencia al deber de coordinar deriva, como es lógico, de la obligación general de consulta. Por lo tanto, para la aplicación de esta excepción, la autoridad debe en primer lugar asegurarse de que las actividades de construcción y mantenimiento de la infraestructura de servicios públicos estén orientadas a beneficiar a los pueblos indígenas. ¿Cómo saberlo? Es obligatorio coordinar previamente con ellos dicha definición. Es decir, es necesaria una actividad de consulta previa para definir el carácter beneficioso de la actividad, y con ello $-y$ las recomendaciones que de ahí surjan- llevar a cabo las correspondientes acciones.

El propio Ministerio de Cultura compartía esta opinión. La Guía Metodológica de la Consulta Previa proveía de criterios para determinar el carácter beneficioso de la medida. Al respecto indicaba que

"Se entenderá que dichos servicios benefician directamente al pueblo indígena:

- Si mejoran la situación socioeconómica de los pueblos indígenas, lo que implica el mejoramiento de las condiciones de vida y de trabajo y del nivel de salud y educación de los pueblos interesados.

- Si no perjudican el bienestar de los pueblos indígenas.

Para determinar dicho beneficio, los/las funcionarios/as estatales deben coordinar de modo previo con los pueblos indígenas, a fin de conocer su posición y lograr acuerdos para la provisión del servicio.

Debe tenerse en cuenta que, si bien en este supuesto no es obligatorio realizar el procedimiento de consulta establecido en el Reglamento, la adopción de las decisiones estatales -en los casos que corresponda- debe considerar la participación de los pueblos indígenas y el pleno respeto de sus derechos colectivos.

Los/as funcionarios/as estatales tendrán en cuenta que los pueblos indígenas podrán solicitar la provisión o mantenimiento de dichos servicios. En este caso, el/la funcionario/a estatal considerará que el servicio público solicitado es beneficioso para dichos pueblos indígenas. 
En cualquier caso, se deben respetar los derechos colectivos y los derechos fundamentales de los pueblos indígenas". 8

Estamos, por lo tanto, frente a la aplicación del principio de flexibilidad de la Ley de Consulta Previa. No es una excepción del deber de consultar -cuestión que no podría ser creada mediante un Decreto Supremo y que tendría que tener base legal- sino de una excepción al uso del procedimiento establecido en el propio reglamento. Desde luego, el Ministerio de Cultura puede emitir lineamientos que orienten la aplicación de este supuesto, de preferencia con la participación de las organizaciones representativas de alcance nacional de los propios pueblos indígenas.

\section{La Directiva 001-2016-VMI/MC y el deber de consultar}

La Directiva 001-2016-VMI/MC ha variado la posición del Ministerio de Cultura arriba reseñada y ha introducido una nueva interpretación de la disposición bajo análisis del Reglamento. La directiva indica que su objetivo es:

"Establecer los procedimientos para la aplicación de lo dispuesto en la Décimo Quinta Disposición Complementaria, Transitoria y Final del Reglamento de la Ley $\mathrm{N}^{\circ} 29785$, en las medidas administrativa vinculadas con la construcción y/o el mantenimiento de la infraestructura en materia de salud o educación, así como la necesaria para la provisión de servicios públicos".

Tras establecer un conjunto de definiciones útiles sobre el concepto de servicios públicos y el contenido del concepto «infraestructura» de los distintos servicios involucrados (salud, educación y servicios públicos), la norma pasa a definir un procedimiento para la aplicación de esta excepción de tres pasos.

1. Determinar el tipo de actividad y el carácter de servicios público. A través de este, la entidad competente para realizar la consulta previa -entidad promotoraidentifica si la propuesta de medida administrativa es una que faculta (título habilitante) la realización de una actividad de construcción o mantenimiento de la infraestructura de los servicios públicos arriba mencionados.

2. Identificar la presencia de pueblos indígenas en el ámbito de las actividades arriba indicadas.

3. Emitir un informe que señale que la medida cumple con los dos supuestos anteriores y que por lo tanto "no corresponde realizar un proceso de consulta previa" ${ }^{\prime \prime}$.

De forma posterior -tras aprobar la medida sin consulta previa- la autoridad que emitió la medida elaborará un «Plan Informativo» (en adelante, el Plan) que deberá

8 Ministerio de Cultura, Consulta a los Pueblos Indígenas: Guía Metodológica, Lima, 2013, pp. 22-23.

9 Acápite 6.1 de la Directiva 001-2016-VMI/MC. 
emitir en un plazo de treinta días hábiles posteriores a la aprobación de la medida, y de forma simultánea al desarrollo de las actividades de construcción o mantenimiento de la infraestructura de los servicios. Es recién con este Plan que se menciona la identificación de los beneficios de la medida, las actividades de coordinación con los pueblos indígenas y el procedimiento de información a llevar a cabo. El Plan luego debe ser ejecutado junto a los pueblos indígenas, debiendo elaborarse tras ello un Informe Final de dicha ejecución, el cual debe ser remitido al Viceministerio de Interculturalidad por la entidad que aprobó la medida que no fue consultada.

Es fácil comprobar que esta directiva no solo va en contra de los principios que la normativa internacional y nacional ha establecido con relación al deber de consultar por parte del Estado, sino que incluso va en contra del texto del Reglamento.

La norma ignora el «beneficio para los pueblos indígenas» como una condición indispensable -y que debe comprobarse previamente- para poder acudir a la excepción procedimental prevista en la Décimo Quinta Disposición Complementaria, Transitoria y Final de Reglamento. Aún más, deja de lado la referencia a que la definición del carácter beneficioso está ligada directamente a la necesidad de coordinar previamente con dichos pueblos. ¿Y si los pueblos indígenas consideran que dicha medida no los beneficia? En segundo lugar, lo que es aún peor, trata el caso no como uno de aplicación del principio de flexibilidad -que llevaría a un procedimiento de consulta más simple y célere- sino a una excepción del deber de consultar, cuestión que es imposible de establecerse por vía reglamentaria, debiendo derivarse de disposiciones de carácter constitucional y legal. La dispensa de consulta de medidas sobre infraestructura no cuenta con dicho respaldo.

Por lo tanto, nos encontramos frente a una directiva contraria a las disposiciones de rango constitucional que han definido el deber de consultar, respaldado además por la Ley de Consulta Previa, así como al propio Reglamento de la Ley de Consulta Previa que buscaría ayudar a aplicar. En consecuencia, podrían interponerse acciones legales en contra de las medidas aprobadas sin consulta -cuando las medidas hayan afectado a los pueblos indígenas, de forma positiva o negativa- derivando eventualmente en la nulidad de las mismas. Del mismo modo, podría interponerse acciones contra la propia Directiva 001-2016-VMI/MC.

No obstante, es probable que en la gran mayoría de casos no se interpongan acciones contra las medidas inconsultas. Esto se debe a que efectivamente las actividades de construcción y mantenimiento de la infraestructura de servicios es apreciada por los pueblos indígenas, y la consideran en general muy beneficiosa. Esto, desde luego, hace más extraña la posición del Ministerio de Cultura.

\section{Alternativas de regulación}


Este caso nos puede llevar a una reflexión más amplia. Gran parte del éxito de la Consulta Previa como política pública deriva de algo más amplio e importante que el derecho y el procedimiento mismo: la construcción de una nueva relación entre los pueblos indígenas y el Estado Peruano. Es evidente que una política que lleve a la realización de miles de procedimientos con efectos insignificantes solo conducirá al agotamiento del proceso y, seguramente, lo presentará ante los ojos de los actores involucrados con una mera formalidad a cumplir. Nada es más dañino para la consulta y para el enfoque intercultural.

¿Existe alguna vía para atender estos casos? Un camino mucho más interesante es uno en el cual las organizaciones representativas de los pueblos indígenas y las autoridades estatales aprueben cada año un plan de desarrollo de la infraestructura de servicios públicos en las tierras indígenas -lo que constituye además una demanda histórica de los pueblos indígenas- definiendo juntos un conjunto de lineamientos para el desarrollo de dicha infraestructura. Esto incluirá medidas de actuación en el caso se produzcan desacuerdos entre las comunidades campesinas o nativas y las autoridades estatales.

Al final, se presentará un informe sobre el desarrollo de estos procesos, pudiendo plantearse sugerencias de mejoras y recomendaciones al respecto. Una salida de este tipo -que puede replicarse en otras áreas de la gestión pública- permitirá mantener los objetivos generales de la consulta previa, en el marco del principio de flexibilidad recogido en la Ley de Consulta Previa. De esta manera, el procedimiento de consulta previa del Reglamento puede reservarse para casos de mayor complejidad. Incluso alguna obra de infraestructura de servicios públicos podría requerirlo, cuestión que también podría preverse en el diálogo previo entre las organizaciones indígenas y las autoridades estatales.

En síntesis, la consulta previa constituye -qué duda cabe- un derecho y a la vez una herramienta clave de la política indígena. Aún hay mucho por aprender de su aplicación efectiva y existe un campo grande de mejora. Para ello, debemos estar atentos más a los objetivos de mayor alcance de la consulta previa y a sus principios, para no terminar cayendo en soluciones que, además de contrarias a la normativa legal, pueden terminar por generar, eventualmente, nuevos conflictos. 